\title{
Bounds on the number of ESSs of a matrix game
}

\author{
M. Broom * \\ Centre for Statistics and Stochastic Modelling, School of Mathematical Sciences, University of Sussex, Falmer, \\ Brighton BN1 9QH, UK
}

Received 24 June 1999; received in revised form 27 June 2000; accepted 3 July 2000

\begin{abstract}
It is well known that for any evolutionary game there may be more than one evolutionarily stable strategy (ESS). In general, the more ESSs there are, the more difficult it is to work out how the population will behave (unless there are no ESSs at all). If a matrix game has an ESS which allows all possible pure strategies to be played, referred to as an internal ESS, then no other ESS can exist. In fact, the number of ESSs possible is highly dependent upon how many of the pure strategies each allow to be played, their support size. It is shown that if $\alpha$ is the ratio of the mean support size to the number of pure strategies $n$, then as $n$ tends to infinity the greatest number of ESSs can be represented by a continuous function $f(\alpha)$ with useful regularity properties, and bounds are found for both $f(\alpha)$ and the value $\alpha^{*}$, where it attains its maximum. Thus we can obtain a limit on the complexity of any particular system as a function of its mean support size. (C) 2000 Elsevier Science Inc. All rights reserved.
\end{abstract}

Keywords: Evolutionarily stable strategies; Payoff; Quadratic form; Complexity; Upper bounds; Lower bound

\section{Introduction}

Game theory has proved a valuable tool for modelling biological populations. The concept of an evolutionarily stable strategy (ESS) (see [1] or [2]) is of particular importance, and is widely applied in considering the strategies adopted by various organisms. In rough terms, an ESS is a strategy which, if played by almost all members of a population, cannot be displaced by a small invading group playing any alternative strategy. Thus an ESS will persist as the dominant strategy through time, so that strategies observed in the real world will tend to be ESSs. For any specific game theoretic model of a real population, simplifying assumptions must be made to obtain

\footnotetext{
${ }^{*}$ Tel.: +44-1273 877 243; fax: +44-1273 678097.

E-mail address: m.broom@sussex.ac.uk (M. Broom).
} 
meaningful results. The games considered in this paper rely on the assumptions that the population reproduces asexually and is both essentially infinite in size and 'well-mixed', so that an animal is equally likely to meet any other animal in a given contest. The extent to which the model predictions are borne out by reality depends, of course, on how serious departures from these assumptions are.

An important class of games is that of matrix games. A matrix game is defined in the following way. Suppose that in a conflict there are $n$ available pure strategies, $S_{1}, \ldots, S_{n}$, and that the payoff to a player playing $S_{i}$ against a player playing $S_{j}$ is $a_{i j}$. The payoffs can thus be written in terms of the payoff matrix $A=\left(a_{i j}\right)$. The Hawk-Dove game of Maynard Smith [1] is an example of such a game.

An individual may play a mixed strategy $\mathbf{p}=\left(p_{1}, p_{2}, \ldots, p_{n}\right)$, where it plays $S_{i}$ with probability $p_{i}$ for all $i$. If a player playing $\mathbf{p}$ meets one playing $\mathbf{q}$, then the payoff to the $\mathbf{p}$-player is

$$
E[\mathbf{p}, \mathbf{q}]=\sum_{i, j} p_{i} q_{j} a_{i j}=\mathbf{p}^{\mathrm{T}} A \mathbf{q}
$$

A strategy $\mathbf{p}$ is evolutionarily stable (ES) against $\mathbf{q}$ if

(i) $\mathbf{p}^{\mathrm{T}} A \mathbf{p} \geqslant \mathbf{q}^{\mathrm{T}} A \mathbf{p}$ and

(ii) if $\mathbf{p}^{\mathrm{T}} A \mathbf{p}=\mathbf{q}^{\mathrm{T}} A \mathbf{p}$ then $\mathbf{p}^{\mathrm{T}} A \mathbf{q}>\mathbf{q}^{\mathrm{T}} A \mathbf{q}$.

$\mathbf{p}$ is an ESS if it is ES against all $\mathbf{q} \neq \mathbf{p}$.

The support of $\mathbf{p}, S(\mathbf{p})$ is defined as the set $\left\{i: p_{i}>0\right\}$. The size of the support $S(\mathbf{p})$ is the number of elements in the support. Matrix games may have multiple ESSs. Following [3], the Pattern of $A$ is the collection of the supports of all the ESSs of the matrix $A$. The problem of which patterns are possible is discussed in [4,5], amongst others.

This problem is of relevance when considering the behaviour of a population of animals split into several habitats. If the habitats are isolated, behaviour may evolve differently in each habitat due to different initial conditions or to random effects, even if the underlying environmental conditions, and so the payoffs they give rise to, are the same. It is thus of interest to consider which strategies are consistent with each other, in the sense of being reachable from the same environmental conditions, and which are incompatible. If we find two strategies which could not have come from the same payoff matrix, we can conclude that different conditions prevail in the separate habitats. An example of when this idea might be useful is when considering the mating strategies adopted by frogs in different pools.

It is well known that if a game has an interal ESS, then no other ESSs can exist. Such a situation is intrinsically simpler than a case where there are a large number of ESSs, each of which could be reached by the population depending upon initial conditions and chance. In fact, the number of ESSs possible is highly dependent upon their support size. The greater the number of ESSs, the more complex the biological situation and the harder it is to understand why animals are behaving in the way that they are. This paper considers the problem of the maximum number of ESSs possible, and thus the potential complexity of the system, as a function of the mean support size of those ESSs.

A related problem was discussed in [6], where the problem of determining the greatest possible number of local maxima that a quadratic form can have when the vector is constrained within the unit simplex was considered. 
Define the vector function

$$
V(\mathbf{p})=\mathbf{p}^{\mathrm{T}} A \mathbf{p},
$$

where $A=\left(a_{i j}\right)$ is a real symmetric $n \times n$ matrix and $\mathbf{p}$ is a probability vector (i.e. its elements are non-negative and sum to 1). The matrix $A$ thus defines a quadratic form in $n$ dimensions. A vector $\mathbf{p}$ is a local maximum of the quadratic form, if and only if it is an ESS of the game with payoff matrix $A$.

This problem arises naturally in the field of population genetics. Suppose that there are $n$-alleles, $A_{1}, \ldots, A_{n}$, at a particular locus and the viability of genotype $A_{i} A_{j}$ is $a_{i j}\left(a_{i j}=a_{j i}\right.$, and so the matrix is symmetric). The allelic frequency $p_{i}$ of $A_{i}$ is given by the following differential equation for each $i$ :

$$
\frac{\mathrm{d} p_{i}}{\mathrm{~d} t}=p_{i}\left((A \mathbf{p})_{i}-\mathbf{p}^{\mathrm{T}} A \mathbf{p}\right) .
$$

If all the $p_{i}$ are initially positive, then the population will converge to a local maximum, which is a locally stable point of the differential equation [7]. Thus the set of local maxima is equivalent to the set of points which the population could conceivably settle at.

This dynamic can also be applied to the game theoretic situation. If the payoff matrix for a game is symmetric, the two situations are identical, otherwise the system's behaviour is more complicated (see [8] or [9] for a discussion of this dynamic).

\section{Results}

For each payoff matrix $A, \mathbf{p}$ is allowed to take values over its entire range, and the ESSs are catalogued. We shall refer to these as the ESSs of $A$. Let $M(A)$ denote the number of ESSs of $A$ and $L(A)$ denote the mean support size of these ESSs.

In [6], $U_{n}$ was defined as the greatest number of local maxima on $n$ dimensions and $u_{n}(r)$ was defined as the greatest number of local maxima of support size $r$ on $n$ dimensions. In this paper, we let $V_{n}$ denote the greatest number of ESSs possible on $n$ strategies and let $v_{n}(r)$ be the greatest number of ESSs of support size $r$ on $n$ strategies.

Further define $s_{n}(x)$ as the greatest number of ESSs of mean support size $x$ on $n$ strategies, so that $s_{n}(x)$ is the maximum of $M(A)$ over $n \times n$ symmetric matrices $A$ with $L(A)=x$.

Sometimes there will be no possible collection of supports with mean size $x$. In this case we set $s_{n}(x)$ to be 0 .

Thus $x$ is not necessarily an integer, but if it is, $s_{n}(x) \geqslant v_{n}(x)$. Clearly $s_{n}(x) \leqslant V_{n}$.

Several results about $s_{n}(x)$ and a related function $f$ will now be given. Proofs of all theorems and corollaries are in Appendix A.

Note that it is fairly easy to show that all our results about ESSs can just as readily be applied to local maxima of constrained quadratic forms.

We now let $\alpha$ be the ratio of the mean support size of a matrix $x$ and the number of strategies $n$. It is shown that the limit of the $n$th root of $s_{n}(\alpha n)$ as $n \rightarrow \infty$ exists; we set this to equal $f(\alpha)$.

$f(\alpha)$ is thus a measure of the potential biological complexity at different values of $\alpha ; \alpha=0$ corresponds to the 'edge' of the strategy space (very small support size), $\alpha=1$ corresponds to the 
'centre' of the space (internal ESS or 'almost internal'). The higher $f(\alpha)$, the more potentially complicated the system is. It is shown in Theorem 2 that $s_{n}(\alpha n) \geqslant 1$ for all sufficiently large $n$, and since no two ESSs can have the same support, $s_{n}(\alpha n) \leqslant 2^{n}$. It is thus clear that $1 \leqslant f(\alpha) \leqslant 2$. We can do a lot better than this, however. It is shown that $f(\alpha)$ has regular properties, and upper and lower bounds for $f(\alpha)$ and $\alpha^{*}$, the value where $f$ reaches its maximum, are found.

\section{Theorem 1.}

$$
s_{m}(x) s_{n}(y) \leqslant s_{m+n}(x+y) .
$$

Thus the number of ESSs possible increases exponentially. In particular $s_{r n}(r y) \geqslant\left(s_{n}(y)\right)^{r}$.

\section{Theorem 2.}

(i) $s_{n}(\alpha n)$ is zero for $\alpha$ not a member of $(0,1]$.

(ii) $s_{n}(\alpha n)$ is zero for non-rational $\alpha$.

(iii) If $\alpha \in(0,1]$ and rational, then $s_{n}(\alpha n)$ is non-zero $\forall n \geqslant N$ for some integer $N$.

Theorems 1 and 2 can now be used to show that the limit as $n$ tends to infinity of $\left(s_{n}(\alpha n)\right)^{1 / n}$ exists for every $\alpha$ between 0 and 1 .

\section{Theorem 3.}

(i) $\operatorname{Lim}_{n \rightarrow \infty} V_{n}^{1 / n}$ exists and is $\leqslant 2$.

(ii) $\operatorname{Lim}_{n \rightarrow \infty}\left(s_{n}(\alpha n)\right)^{1 / n}(=f(\alpha))$ exists $\forall$ rational $\alpha \in(0,1]$, and $\left.s_{n}(\alpha n)\right)^{1 / n} \leqslant f(\alpha) \forall n$.

Furthermore, the function $f$ is shown to have a number of regular properties. It is continuous, its logarithm is concave, it possesses left- and right-derivatives (except at the end points 0 and 1) and is bounded above and below. Specific upper and lower bounds for $f(\alpha)$ are given.

\section{Theorem 4.}

$$
(f(\alpha))^{1-y}(f(\beta))^{y} \leqslant f(\alpha(1-y)+\beta y), \quad \alpha, \beta, y \in[0,1], \text { and rational. }
$$

Theorem 4 implies that

$$
(1-y) \ln (f(a))+y \ln (f(\beta)) \leqslant \ln f(\alpha(1-y)+\beta y), \quad y \in[0,1],
$$

i.e. that $\ln f$ is a concave function.

Corollary 1. $f(\alpha)$ is continuous on the set of rationals in $[0,1]$.

Hence we can define $f(\alpha)=\lim f\left(\alpha_{j}\right)$ for any non-rational $\alpha$ between zero and one, inclusive, where $\left\{\alpha_{j}\right\}$ is a sequence of rationals which converges to $\alpha$. Thus $f(\alpha)$ is defined for every real $\alpha$ in $[0,1]$, and $\ln f(\alpha)$ is concave on the reals in $[0,1]$.

Corollary 2. The right- and left-derivatives of $f(\alpha), f^{\prime}\left(\alpha_{+}\right)$and $f^{\prime}\left(\alpha_{-}\right)$, exist for all $\alpha \in(0,1)$ (the right-derivative does not exist at 0 and the left-derivative does not exist at 1). 
It is not known whether the right- and left-derivatives are equal for all values of $\alpha$.

It also follows that $\ln f(\alpha)$ (and hence $f(\alpha)$ ) cannot have more than one local maximum, since if $\alpha$ and $\beta$ were such maxima then the value of $\ln f$ is at least as great as either $\ln f(\alpha)$ or $\ln f(\beta)$ for all points between $\alpha$ and $\beta$, so both cannot be local maxima. Hence we have a contradiction, i.e. $\ln f$ (and $f$ ) has either a single maximum or a plateau of maxima.

\section{Theorem 5.}

(i) $f(\alpha)$ is bounded above by $1 /\left\{\alpha^{\alpha}(1-\alpha)^{(1-\alpha)}\right\}$.

(ii) $f(\alpha)$ is bounded below by
(a) $1 / \alpha^{\alpha}: \alpha=1 / i$, integer $i$
and by
(b) $(2 /(1-\alpha))^{(1-\alpha) / 2}: \alpha=1-2 / i$, integer $i$.

(iii) $f(0)=f(1)=1$.

Due to the property that $\ln f(\alpha)$ is concave, we can construct a lower bound over all values of $\alpha$, from (ii).

Note that there is a pattern with 30 ESSs (also local maxima, since the matrix with this pattern is symmetric) of support size 3 on 9 strategies (see [6]) giving

$$
f(1 / 3) \geqslant 30^{1 / 9}=1.459,
$$

and similarly there is a pattern with 14 ESSs of support size 3 on 7 strategies [10], so that

$$
f(3 / 7) \geqslant 14^{1 / 7}=1.458 .
$$

It is now possible to use the properties of $f(\alpha)$ together with its upper and lower bounds to obtain bounds upon the value of $\alpha$ which gives the greatest number of ESSs.

Theorem 6. If $\alpha^{*}$ is a value of $\alpha$ for which $f(\alpha)$ attains its maximum, then

(i) $1+\alpha^{*} \leqslant f\left(\alpha^{*}\right) \leqslant 1 /\left(1-\alpha^{*}\right)$,

(ii) $0.315 \leqslant \alpha^{*} \leqslant 0.752$.

Thus the average support size of the most complicated situations is between $31 \%$ and $75 \%$ of the total number of available strategies. If we consider the set of all possible strategies as a vector space, the strategy space, the $i$ th element of which is $p_{i}$, then this implies that the most complex behaviour takes place at the fringes of the strategy space where at least $25 \%$ of dimensions are unused in every ESS.

\section{Examples}

We now consider three examples of questions which can be answered using the above methodology to illustrate the results.

(a) Find bounds on the number of ESSs of mean support size 6 when the total number of strategies is 90 . 
Using Theorems 3 and 5 we know that

$$
1 /\left\{\alpha^{\alpha}(1-\alpha)^{(1-\alpha)}\right\} \geqslant f(\alpha) \geqslant\left(s_{n}(\alpha n)\right)^{1 / n} .
$$

Setting $n=90$ and $\alpha=1 / 15$, we obtain $s_{90}(6) \leqslant(1.27753)^{90}=3.745 \times 10^{9}$. From Theorem 5, since $\alpha=1 / i$, where $i$ is an integer, we have $s_{90}(6) \geqslant\left(1 / \alpha^{\alpha}\right)^{n}=(1.19786)^{90}=1.139 \times 10^{7}$.

(b) Find bounds on $\ln \left[s_{n}(\alpha n)\right]$ for general $n$ and $\alpha=1 / i$, where $i$ is integer valued.

General upper and lower bounds for $\ln \left[s_{n}(\alpha n)\right]$ can be found using Theorem 5 , so that if $\alpha=1 / i$ and $\alpha n$ is integer-valued, then

$$
-n \alpha \ln (\alpha) \leqslant \ln \left[s_{n}(\alpha n)\right] \leqslant-n[\alpha \ln (\alpha)+(1-\alpha) \ln (1-\alpha)] .
$$

Thus when $\alpha=1 / 15$, we have $0.1805 n \leqslant \ln \left[s_{n}(\alpha n)\right] \leqslant 0.2449 n$.

(c) Find bounds on $f(0.16)$.

The upper bound is found as usual from Theorem 5 , giving $f(0.16) \leqslant 1.5522$. The lower bound is found as follows:

$1 / 6 \geqslant 0.16>1 / 7 ; f(1 / 6) \geqslant 1.3480, f(1 / 7) \geqslant 1.3205$ using Theorem 5 .

$0.16=0.72(1 / 6)+0.28(1 / 7)$, so that using Theorem 4 we obtain

$$
f(0.16) \geqslant f(1 / 7)^{0.28} f(1 / 6)^{0.72} \geqslant 1.3402 .
$$

\section{Discussion}

For real populations it is possible that, for given environmental conditions, there may be many ESSs. For any particular situation it is generally worthwhile finding all possible ESSs (although some may be far more likely to occur in practice than others). However it is also of value to consider the problem from the reverse direction. Which situations can generate only a single ESS, which is then the only stable solution, and which are very complicated with multiple ESSs? In particular, for the important class of matrix games, there is a link between the complexity of the system, and the average number of pure strategies in an ESS's support.

It has been shown that the greatest number of ESSs of mean support size $x$ that a matrix game with $n$ strategies may have increases essentially as a constant to the power $n$, as $n$ tends to infinity with $x / n=\alpha$ fixed. This constant rate of increase has been defined as $f(\alpha)$. Thus $f$ is a measure of the potential complexity of a system, as a function of the proportion $\alpha$. The function $f(\alpha)$ has been shown to have some useful regularity properities; it is bounded above and below, is continuous and $\ln f(\alpha)$ is concave. Lower and upper bounds have been found for $f(\alpha)$, and lower and upper bounds have also been found for the value of $\alpha, \alpha^{*}$, which maximises $f(\alpha)$.

It is not surprising that $f(\alpha)$ possesses the regular properties that it does, and it seems reasonable to conjecture that it is differentiable everywhere except at $\alpha=0$ and $\alpha=1$. However it is interesting to be able to show these properties given the apparently limited information available (the detailed study of patterns of ESSs has generally considered those with a low number of strategies only $(\leqslant 5$, see $[3]))$.

It has been shown that the greatest number of ESSs do not occur when these are central, in the sense of involving all pure strategies, or nearly so. In particular $\alpha^{*}$ is less than 0.752 and so the 
ESSs of the most compicated games are peripheral in terms of the strategy space, with about $25 \%$ of strategies not used in each ESS. Thus all of the interesting behaviour takes place at the fringes, rather than in the centre of the space.

The distance between the upper and lower bounds for $f(\alpha)$ is large for some values of $\alpha$. Although it is known that $f(0)=f(1)=1$, the value of $f(1 / 2)$ may be anywhere between $\sqrt{2}$ and 2 . A better lower bound has been obtained at $\alpha=1 / 3$ by finding a payoff matrix for 9 strategies with 30 ESSs, so that we know that $f(1 / 3)$ lies between 1.459 and 1.890 . The lower bound could generally be improved by finding special case patterns. The upper bound could be reduced if extra restrictions were found upon which patterns of ESSs were possible.

In real populations, the number of observed alleles/strategies tends to be relatively small compared to those 'available' (e.g. by mutation), especially when the number of strategies is large, see $[11,12]$. Thus the support sizes that you would expect would be small, giving a low value of $\alpha$, so that the more complicated cases are unlikely to occur in reality.

If we have found some ESSs for a population, and know roughly the number of possible strategies (e.g. the number alleles at a given locus), then we can find an estimate of $\alpha$ and estimate $f(\alpha)$, thus giving an idea of the complexity of the situation that we face. Maybe all the possible ESSs have been found, or possibly there are many more ESSs undiscovered. A knowledge of how $f(\alpha)$ varies with $\alpha$ can provide a useful insight into this problem.

\section{Appendix A}

Proof of Theorem 1. In [6] it is shown that if the collection of ESSs of the $m \times m$ matrix $A$ is $\left\{\mathbf{p}_{1}, \ldots, \mathbf{p}_{k}\right\}$, and the collections of ESSs of the $n \times n$ matrix $B$ is $\left\{\mathbf{q}_{1}, \ldots, \mathbf{q}_{1}\right\}$, then there is a $(m+n) \times(m+n)$ matrix $C$ with $\operatorname{ESSs} \mathbf{r}_{i j} i=1, \ldots, k ; j=1, \ldots, l$, where $\mathbf{r}_{i j}^{\mathrm{T}}=\left(\beta \mathbf{p}_{i}^{\mathrm{T}},(1-\beta) \mathbf{q}_{j}^{\mathrm{T}}\right)$ for some $0<\beta<1$.

Thus the size of $S\left(\mathbf{r}_{i j}\right)$ is equal to the sum of the sizes of $S\left(\mathbf{p}_{i}\right)$ and $S\left(\mathbf{q}_{j}\right)$, and it follows that the mean support size of $C$ is equal to the mean support size of $A$ plus the mean support size of $B$.

If $A$ has $s_{m}(x)$ ESSs of mean support size $x$, and $B$ has $s_{n}(y)$ ESSs of mean support size $y$, then $C$ has $s_{m}(x) s_{n}(y)$ ESSs of mean support size $x+y$ and so the stated inequality then follows immediately.

If either of $s_{n}(x)$ or $s_{m}(y)$ is zero, then clearly the inequality is satisfied.

Proof of Theorem 2. The proof of (i) is trivial. No support can be of size less than one or greater than $n$. Therefore the mean support size $\alpha n$ satisfies

$$
1 \leqslant \alpha n \leqslant n \quad \Rightarrow \quad \frac{1}{n} \leqslant \alpha \leqslant 1
$$

(ii) For a finite number of strategies $n$ there can only be a finite number of $\operatorname{ESSs}\left(s_{n}(x) \leqslant 2^{n}\right)$. Thus for any matrix $A$, $\alpha$ must be rational and therefore $\alpha$ must also be rational.

(iii) Suppose that $\alpha$ is a rational between 0 and 1 . Let $\alpha=l / m ; l$ and $m$ are integers such that $l<m$ (if $l=m$ then a single support size $n$ gives a quadratic form with mean size $\alpha n$ ).

Consider $n=k m$, then choose a single support of size $k l$, and we have a pattern with mean size $k l=(l / m) k m=\alpha n$. Thus there is at least one pattern with mean support size $\alpha n$ and so $s_{n}(\alpha n)$ is non-zero. In particular $s_{n}(\alpha n)$ is non-zero when $n=m^{2} l$. 
The following result is shown in [5]:

Suppose that $A$ is an $n \times n$ matrix such that $a_{i i}=0 \forall_{i}$ and, if $i \neq j$, then $a_{i j}=a_{j i}= \pm 1$. Then the set $S=\left(i_{1}, i_{2}, \ldots, i_{k}\right)$ is the support of an ESS (local maximum) if and only if $a_{i_{l} i_{m}}=1,1 \leqslant l<m \leqslant k$ and no superset of $S$ has this property. Such matrices are referred to as clique matrices. This result will be used to prove (iii).

Now let $n=m^{2} l+j$ for some integer $j, 0<j<m$.

Consider the following matrix A for $n$ strategies:

$$
\mathbf{A}=\left[\begin{array}{llll}
\mathbf{A}_{11} & \mathbf{A}_{12} & \mathbf{A}_{13} & \mathbf{A}_{14} \\
\mathbf{A}_{21} & \mathbf{A}_{22} & \mathbf{A}_{23} & \mathbf{A}_{24} \\
\mathbf{A}_{31} & \mathbf{A}_{32} & \mathbf{A}_{33} & \mathbf{A}_{34} \\
\mathbf{A}_{41} & \mathbf{A}_{42} & \mathbf{A}_{43} & \mathbf{A}_{44}
\end{array}\right]
$$

The submatrix $\mathbf{A}_{i j}$ is $b_{i} \times b_{j}$, where

$$
b_{1}=m l^{2}-1, \quad b_{2}=j l+1, \quad b_{3}=m-1, \text { and } b_{4}=n-b_{1}-b_{2}-b_{3}>0, \quad(m>j, m>l) .
$$

The entries in the submatrices are as follows:

$\mathbf{A}_{i i}$ has 0s down the leading diagonal together with either 1 s everywhere else $(i=1$ or 2$)$, or -1 s everywhere else $(i=3$ or 4$)$.

All entries in $\mathbf{A}_{12}, \mathbf{A}_{21}, \mathbf{A}_{13}$ and $\mathbf{A}_{31}$ are $1 \mathrm{~s}$ and all entries in all of the other submatrices are $-1 \mathrm{~s}$.

A then has one clique ESS of size $m l^{2}+j l$ (comprising the first $m l^{2}+j l$ strategies) and $m-1$ clique ESS of size $m l^{2}$ (containing the first $m l^{2}-1$ strategies together with each of $\left.b_{1}+b_{2}+i, i=1, \ldots, b_{3}\right)$. The mean support size is therefore

$$
\frac{1}{m}\left((m-1) m l^{2}+m l^{2}+j l\right)=m l^{2}+\frac{j l}{m}=\frac{l}{m}\left(m^{2} l+j\right)=\alpha n .
$$

Thus $s_{n}(\alpha n)$ exists for $n=m^{2} l+j$.

If we have a pattern on $k_{1}$ strategies with mean support size $\alpha k_{1}$ and a pattern on $k_{2}$ strategies with mean support size $\alpha k_{2}$ then Theorem 1 shows that there is a pattern on $k_{1}+k_{2}$ strategies with mean support size $\alpha\left(k_{1}+k_{2}\right)$, i.e. if $s_{n}(\alpha n)$ is non-zero for $n=k_{1}$ and $n=k_{2}$ then it is non-zero for $n=k_{1}+k_{2}$.

Now $s_{n}(\alpha n)$ is non-zero for

$$
n=m^{2} l+j \quad \forall 0<j<m, \text { and for } n=k m \quad \forall k .
$$

Hence $s_{n}(\alpha n)$ is non-zero for all $n \geqslant m^{2} l$. This completes the proof of Theorem 2 .

\section{Proof of Theorem 3.}

(i) In [6] it was shown that the limit of $U_{n}^{1 / n}$ as $n$ tends to infinity exists and is equal to $\gamma \leqslant 2$. The identical proof can also be used to show Theorem 3 part (i).

(ii) Suppose that $N$ is a value for which $s_{n}(\alpha n)$ is non-zero for all $n$ greater than $N$.

Define $f_{k}$ by

$$
f_{k}=\frac{\left(s_{k N}(\alpha k N)\right)^{1 / N}}{\left(s_{N}(\alpha N)\right)^{1 / N}} \Rightarrow f_{1}=1
$$




$$
\begin{aligned}
f_{k} f_{l} & =\frac{\left(s_{k N}(\alpha k N)\right)^{1 / N}}{\left(s_{N}(\alpha N)\right)^{1 / N}} \frac{\left(s_{l N}(\alpha l N)\right)^{1 / N}}{\left(s_{N}(\alpha N)\right)^{1 / N}} \\
& =\frac{\left(s_{k N}(\alpha k N) s_{l N}(\alpha l N)\right)^{1 / N}}{\left(s_{N}(\alpha N)\right)^{1 / N}} \frac{1}{\left(s_{N}(\alpha N)\right)^{1 / N}} \\
& \leqslant \frac{1}{\left(s_{N}(\alpha N)\right)^{1 / N}} \frac{\left(s_{(k+l) N}(\alpha(k+l) N)\right)^{1 / N}}{\left(s_{N}(\alpha N)\right)^{1 / N}} \\
& =\frac{f_{k+l}}{\left(s_{N}(\alpha N)\right)^{1 / N}} \\
& \leqslant f_{k+l} .
\end{aligned}
$$

by Theorem 1 . Since $V_{n} \leqslant 2^{n}$,

$$
s_{k N}(\alpha k N) \leqslant 2^{k N} \Rightarrow f_{k} \leqslant 2^{k} .
$$

Hence by a result of [13] $f_{k}^{1 / k}$ converges.

Now consider $n=k N+r$, where $0<r<N$ :

$$
s_{n}(\alpha n)=s_{k N+r}(\alpha(k N+r)) \geqslant s_{(k-1) N}(\alpha(k-1) N) s_{N+r}(\alpha(N+r)) \geqslant s_{(k-1) N}(\alpha(k-1) N) .
$$

Similarly

$$
s_{(k+2) N}(\alpha(k+2) N) \geqslant s_{k N+r}(\alpha(k N+r)) s_{2 N-r}(\alpha(2 N-r)) \quad \Rightarrow \quad s_{n}(\alpha n) \leqslant s_{(k+2) N}(\alpha(k+2) N) .
$$

Thus

$$
\begin{aligned}
{\left[s_{(k-1) N}(\alpha(k-1) N)\right]^{1 /((k+1) N)} } & \leqslant\left[s_{(k-1) N}(\alpha(k-1) N)\right]^{1 / n} \\
& \leqslant\left[s_{n}(\alpha n)\right]^{1 / n} \\
& \leqslant\left[s_{(k+2) N}(\alpha(k+2) N)\right]^{1 / n} \\
& \leqslant\left[s_{(k+2) N}(\alpha(k+2) N)\right]^{1 / k N} \\
& \Rightarrow\left[f_{k-1}^{1 /(k-1)}\right]^{(k-1) /(k+1)}\left[s_{N}(\alpha N)\right]^{1 / N} \\
& \leqslant\left[s_{n}(\alpha n)\right]^{1 / n} \\
& \leqslant\left[f_{k+2}^{1 /(k+2)}\right]^{(k+2) / k}\left[s_{N}(\alpha N)\right]^{1 / N} .
\end{aligned}
$$

Thus the sequence $\left[s_{n}(\alpha n)\right]^{1 / n}$ is squeezed between two sequences which both converge to the limit of $\left.\left[s_{k N}(\alpha k N)\right]^{1 / k N}\right]$. This means that the limit of the sequence with $n=k N+r$ is the same for all values of $r=0,1, \ldots, N-1$, i.e. $\left[s_{n}(\alpha n)\right]^{1 / n}$ converges for any rational $\alpha$.

Finally

$$
\left[s_{k n}(\alpha k n)\right]^{1 / k n} \geqslant\left[s_{n}(\alpha n)\right]^{1 / n} \quad \forall k
$$

and so $\left[s_{n}(\alpha n)\right]^{1 / n} \leqslant f(\alpha)$. 


\section{Proof of Theorem 4.}

$$
s_{n}((\alpha(1-y)+\beta y) n) \geqslant s_{n y}(\beta n y) s_{n(1-y)}(\alpha(1-y) n),
$$

where $\alpha, \beta$ and $y$ are all rationals between 0 and 1 . Thus

$$
\begin{aligned}
\left(s_{n}((\alpha(1-y)+\beta y) n)\right)^{1 / n} & \geqslant\left(s_{n y}(\beta n y)\right)^{1 / n}\left(s_{n(1-y)}(\alpha n(1-y))\right)^{1 / n} \\
& =\left[\left(s_{n y}(\beta n y)\right)^{1 / y n}\right]^{y}\left[\left(s_{n(1-y)}(\alpha n(1-y))\right)^{1 /(1-y) n}\right]^{1-y} .
\end{aligned}
$$

Letting $n$ tend to infinity (over values where $n y$ is an integer only), the above expression implies that

$$
f(\alpha(1-y)+\beta y) \geqslant f(\beta)^{y} f(\alpha)^{1-y}
$$

for any rational value of $y$.

Proof of Corollary 1. $\ln f(\alpha)$ is a concave function, which implies that it is continuous by a result of [14]. Thus $f(\alpha)$ is also continuous.

Proof of Corollary 2. The existence of the derivatives in the interior of the range of $\alpha$ follows immediately from the fact that $f(\alpha)$ is concave (for example, see Ref. [14]).

It is easy to show that the right-derivative does not exist at 0 and that the left-derivative does not exist at 1 by considering the upper and the lower bounds given in Theorem 5. $f(\alpha)$ is squeezed between two functions whose derivative tends to $\infty$ (at 0 ) and to $-\infty$ (at 1 ), so that the derivative of $f(\alpha)$ is likewise; hence the derivative does not exist at 0 or 1 .

Proof of Theorem 5. (i) Suppose that we have a pattern on $n$ strategies with mean support size $r$. Suppose further that the number of supports of size $i$ is $p_{i}$, so that the total number of supports is $\sum p_{i}$ and, since the set of supports must form an anti-chain [16], it is shown in [15] that

$$
\sum p_{i} \frac{i !(n-i) !}{n !} \leqslant 1 \ldots(2) \text {. }
$$

It is easy to show that for any $k<l$,

$$
\frac{k !(n-k) !}{n !}+\frac{l !(n-l) !}{n !} \geqslant \frac{(k+1) !(n-k-1) !}{n !}+\frac{(l-1) !(n-l+1) !}{n !} .
$$

Suppose that $k<r<l, p_{k}>0$ and is the smallest value for which this is true and $p_{l}>0$ and is the largest value for which this is true. If we replace values of $p_{i}$ as follows:

$$
\begin{aligned}
& p_{k} \rightarrow p_{k}-1, \quad p_{k+1} \rightarrow p_{k+1}+1, \\
& p_{l-1} \rightarrow p_{l-1}+1, \quad p_{l} \rightarrow p_{l}-1,
\end{aligned}
$$

then $\sum p_{i}$ remains the same, and the sum in (2) decreases. Eventually the only non-zero $p_{i}$ is when $i=r$, and so

$$
\sum p_{i} \frac{r !(n-r) !}{n !} \leqslant 1 \Rightarrow \sum p_{i} \leqslant \frac{n !}{r !(n-r) !} .
$$


Letting $r=\alpha n$, we get

$$
s_{n}(\alpha n) \leqslant \frac{n !}{\alpha n !((1-\alpha) n) !} .
$$

For large $m, m ! \cong(2 \pi m)^{1 / 2}(m / e)^{m}$.

In particular $0.5(2 \pi m)^{1 / 2}(m / e)^{m}<m !<2(2 \pi m)^{1 / 2}(m / e)^{m}$.

$$
\begin{gathered}
\Rightarrow \frac{n !}{\alpha n !((1-\alpha) n) !}<8 \frac{(2 \pi n)^{1 / 2}}{(2 \pi \alpha n)^{1 / 2}(2 \pi(1-\alpha) n)^{1 / 2}} \frac{(n / e)^{n}}{(\alpha n / e)^{\alpha n}((1-\alpha) n / e)^{(1-\alpha) n}} \\
=\frac{8}{(2 \pi \alpha(1-\alpha) n)^{1 / 2}}\left(\frac{1}{\alpha^{\alpha}(1-\alpha)^{(1-\alpha)}}\right)^{n} \\
\Rightarrow\left(s_{n}(\alpha n)\right)^{1 / n} \leqslant\left(\frac{8}{(2 \pi \alpha(1-\alpha) n)^{1 / 2}}\right)^{1 / n} \frac{1}{\alpha^{\alpha}(1-\alpha)^{(1-\alpha)}} \rightarrow \frac{1}{\alpha^{\alpha}(1-\alpha)^{(1-\alpha)}} .
\end{gathered}
$$

(ii) Let $n=k i$. Then there is an $n \times n$ clique matrix which has $i^{k}$ ESSs with supports of size $k$ [5]. Thus the mean support size is $n / i$ and so $\alpha=1 / i$, and the number of supports is

$$
i^{n / i}=\left(\frac{1}{\alpha}\right)^{\alpha n} \text {, }
$$

so that

$$
s_{n}(\alpha n) \geqslant\left(\frac{1}{\alpha}\right)^{\alpha n} \text {. }
$$

Letting $n$ tend to infinity, we get

$$
f(\alpha) \geqslant\left(\frac{1}{\alpha}\right)^{\alpha}, \quad \alpha=\frac{1}{i} .
$$

Now let $\alpha=1-2 / i$, so that for $i$ strategies, $\alpha i=i-2$. In [6] it is shown that there is a circulant matrix on $i$ strategies which has $i$ ESSs each with support size $i-2$. So for $n=k i$ strategies, there is a matrix with $i^{k}$ supports of size $\alpha n$ (using Theorem 2). Thus

$$
s_{n}(\alpha n) \geqslant\left(\frac{2}{1-\alpha}\right)^{n(1-\alpha) / 2} \Rightarrow f(\alpha) \geqslant\left(\frac{2}{1-\alpha}\right)^{(1-\alpha) / 2}
$$

for $\alpha=1-2 / i$.

(iii)

$$
\begin{aligned}
& f(1)=\lim _{n \rightarrow \infty}\left[s_{n}(n)\right]^{1 / n}=1, \quad\left(s_{n}(n)=1 \forall n\right) \\
& f(0)=\lim _{\alpha \rightarrow 0} f(\alpha) \leqslant \lim _{\alpha \rightarrow 0} \frac{1}{\alpha^{\alpha}(1-\alpha)^{(1-\alpha)}}=1 .
\end{aligned}
$$

Clearly $f(\alpha) \geqslant 1$ for any $\alpha$, i.e. $f(0)=1$.

Note that in [5] it is shown that, when $n=r+q k$, a matrix can be found with

$$
q^{k-r}(q+1)^{r}
$$


supports, and a general lower bound for $f(\alpha)$ for all rational $\alpha$ can be obtained from this. This turns out to be precisely the same lower bound as we obtained above when $\alpha \leqslant 0.5$, and to be lower than our lower bound for higher $\alpha$.

Proof of Theorem 6. (i) Suppose that there is a unique value of $\alpha$ which gives a maximum value for $f(\alpha)$.

Consider a pattern on $n-1$ strategies which attains the maximum number of supports $V_{n-1}$. Suppose that its mean support size is $x_{n-1}$.

The following result is shown in [17]:

Suppose that $A$ is an $n \times n$ matrix and the pattern of $A$ is $\left\{T_{1}, \ldots, T_{m}, T_{m+1}, \ldots, T_{k}\right\}$, where $i \in T_{j}$ if and only if $j \leqslant m$. Then there is an $(n+1) \times(n+1)$ matrix with pattern $\left\{T_{1}, \ldots, T_{m}, T_{m+1}, \ldots, T_{k}, T_{1}^{\prime}, \ldots, T_{m}^{\prime}\right\}$, where $T_{j}^{\prime}=T_{j} /(i) \cup(n+1)$.

The above implies that the element appearing most commonly in the supports can be 'split' to give a new pattern with at least $V_{n-1} x_{n-1} /(n-1)$ extra supports. Thus

$$
V_{n-1} \frac{n-1+x_{n-1}}{n-1} \leqslant V_{n} .
$$

Now consider a pattern on $n$ strategies which has $V_{n}$ supports. Suppose that its mean support size is $x_{n}$. It is shown in [6] that we can 'remove' the element appearing least commonly in the supports to give a new pattern with at most $V_{n} x_{n} / n$ less supports. Thus

$$
V_{n} \leqslant V_{n-1} \frac{n}{n-x_{n}} .
$$

Considering a sequence of such patterns, we have that

$$
V_{n-k} \prod_{i=n-k}^{n-1} \frac{i+x_{i}}{i} \leqslant V_{n} \leqslant V_{n-k} \prod_{i=n-k+1}^{n} \frac{i}{i-x_{i}},
$$

setting $k=n / 2$ and taking powers of $2 / n$, we obtain

$$
V_{n / 2}^{2 / n}\left(\prod_{i=n / 2}^{n-1} \frac{i+x_{i}}{i}\right)^{2 / n} \leqslant\left(V_{n}^{1 / n}\right)^{2} \leqslant V_{n / 2}^{2 / n}\left(\prod_{i=(n / 2)+1}^{n} \frac{i}{i-x_{i}}\right)^{2 / n} .
$$

Letting $n$ tend to infinity in the above expression, $x_{i} / i \rightarrow \alpha^{*}$ and so we obtain

$$
1+\alpha_{*} \leqslant \gamma \leqslant \frac{1}{1-\alpha_{*}} .
$$

If there is not a unique value of $\alpha$ for which $f(\alpha)=\gamma$, then the above procedure must be followed for every $\alpha$, using a series of patterns which have $\left(x_{n} / n\right)$ converging to $\alpha$ and number of supports which when put to the power $1 / n$ converges to $\gamma$.

Thus the above inequality is true for any such $\alpha^{*}$.

(ii) We have from part (i) that $f\left(\alpha^{*}\right) \leqslant 1 /\left(1-\alpha^{*}\right)$. We know that $f(1 / 3) \geqslant 30^{1 / 9}$, so that $1 /\left(1-\alpha^{*}\right) \geqslant 30^{1 / 9} \Rightarrow \alpha^{*} \geqslant 0.3147$. 
We also know that $f\left(\alpha^{*}\right) \geqslant 1+\alpha^{*}$. Now

$$
f(\alpha) \leqslant \frac{1}{\alpha^{\alpha}(1-\alpha)^{(1-\alpha)}} \quad \Rightarrow \quad 1+\alpha^{*} \leqslant f\left(\alpha^{*}\right) \leqslant \frac{1}{\alpha^{* \alpha^{*}}\left(1-\alpha^{*}\right)^{\left(1-\alpha^{*}\right)}} .
$$

The above inequality can be solved iteratively, and gives $\alpha^{*} \leqslant 0.7516$.

\section{References}

[1] J. Maynard Smith, Evolution and the Theory of Games, Cambridge University, New York, 1982.

[2] J. Maynard Smith, G.R. Price, The logic of animal conflict, Nature 246 (1973) 15.

[3] G.T. Vickers, C. Cannings, Patterns of ESS's 1, J. Theoret. Biol. 132 (1988) 387.

[4] M. Broom, C. Cannings, G.T. Vickers, Patterns of ESSs: adding pairs to an ESS, Math. Biosci. 136 (1997) 21.

[5] C. Cannings, G.T. Vickers, Patterns of ESS's 2, J. Theoret. Biol. 132 (1988) 409.

[6] M. Broom, C. Cannings, G.T. Vickers, On the number of local maxima of a constrained quadratic form, Proc. Roy. Soc. London A 443 (1993) 573.

[7] J.F.C. Kingman, A matrix inequality, Quart. J. Math. 12 (1961) 78.

[8] J. Hofbauer, K. Sigmund, The Theory of Evolution and Dynamical Systems, Cambridge University, New York, 1988.

[9] R. Cressman, The stability concept of evolutionary game theory: a dynamic approach, Lecture Notes Biomath.94, Springer, Berlin, 1992.

[10] G.T. Vickers, C. Cannings, On the number of stable equilibria in a one-locus multi-allelic system, J. Theoret. Biol. 131 (1988) 273.

[11] J.F.C. Kingman, Typical polymorphisms maintained by selection at a single locus, J. Appl. Prob. 25A (1988) 113.

[12] J. Haigh, How large is the support of an ESS? J. Appl. Prob. 26 (1989) 164.

[13] G. Polya, G. Szego, Problems and Theorems in Analysis, Springer, Berlin, vol. 1, 1972 (problem 98).

[14] M. Spivak, Calculus, Addison-Wesley, Reading, MA, 1967, p. 199 (problems 9 and 10).

[15] E. Sperner, Ein Satz uber Untermenge einer endlichen Menge, Math. Z. 27 (1928) 544.

[16] D.T. Bishop, C. Cannings, Models of animal conflict, Adv. Appl. Prob. 8 (1976) 616.

[17] M. Broom, C. Cannings, G.T. Vickers, Sequential methods in patterns of ESSs, J. Math. Biol 32 (1994) 597. 